\section{A temporal requirement for Hippo signaling in mammary gland differentiation, growth, and tumorigenesis}

\author{
Qian Chen, ${ }^{1}$ Nailing Zhang, ${ }_{1}^{1}$ Ryan S. Gray, ${ }^{2}$ \\ Huili Li, ${ }^{3}$ Andrew J. Ewald, ${ }^{2,3}$ \\ Cynthia A. Zahnow, ${ }^{3,4}$ and Duojia Pan $^{1,4}$ \\ ${ }^{1}$ Department of Molecular Biology and Genetics, Howard \\ Hughes Medical Institute, ${ }^{2}$ Department of Cell Biology, \\ ${ }^{3}$ Department of Oncology, Sidney Kimmel Comprehensive \\ Cancer Center, Johns Hopkins University School of Medicine, \\ Baltimore, Maryland 21205, USA
}

Despite recent progress, the physiological role of Hippo signaling in mammary gland development and tumorigenesis remains poorly understood. Here we show that the Hippo pathway is functionally dispensable in virgin mammary glands but specifically required during pregnancy. In contrast to many other tissues, hyperactivation of YAP in mammary epithelia does not induce hyperplasia but leads to defects in terminal differentiation. Interestingly, loss of YAP causes no obvious defects in virgin mammary glands but potently suppresses oncogeneinduced mammary tumors. The selective requirement for YAP in oncogenic growth highlights the potential of YAP inhibitors as molecular targeted therapies against breast cancers.

Supplemental material is available for this article.

Received October 24, 2013; revised version accepted January 27, 2014.

The Hippo tumor suppressor pathway acts through a kinase cascade to inhibit the transcriptional coactivator Yorkie (Yki) in Drosophila or its mammalian homologs, YAP and TAZ (Pan 2010; Zhao et al. 2010; Halder and Johnson 2011). This pathway was initially discovered in Drosophila for its critical role in restricting imaginal disc growth by promoting cell cycle exit and apoptosis. More recent studies have implicated Hippo signaling as a conserved regulator of organ size, tissue regeneration, and stem cell biology. The importance of Hippo signaling is further underscored by the widespread activation/ overexpression of the YAP oncoprotein in human cancers.

Despite recent progress in understanding Hippo signaling in mammalian development, the physiological roles of the Hippo pathway in many mammalian tissues remain unexplored. Of particular interest to this study is the mammary gland. Although several studies have im-

[Keywords: Hippo signaling; YAP; differentiation; mammary gland; proliferation; tumorigenesis]

${ }^{4}$ Corresponding authors

E-mail djpan@jhmi.edu

E-mail zahnoci@jhmi.edu

Article is online at http://www.genesdev.org/cgi/doi/10.1101/gad.233676.113. plicated dysregulation of the Hippo pathway in breast cancers (Overholtzer et al. 2006; Chan et al. 2008; Lei et al. 2008; Cordenonsi et al. 2011), its physiological role in mammary gland development is unknown. Even in breast tumorigenesis, the contribution of Hippo signaling is controversial. For example, the Yap locus was identified as an amplicon in murine mammary tumors derived from a Brca1/p53 mutant background (Overholtzer et al. 2006), and YAP/TAZ overexpression was detected in a significant fraction of primary breast cancers (Chan et al. 2008; Cordenonsi et al. 2011). Consistent with an oncogenic function, YAP/TAZ can promote epithelial-to-mesenchymal transition (Overholtzer et al. 2006; Chan et al. 2008), metastasis (Chen et al. 2012; Lamar et al. 2012), and selfrenewal (Cordenonsi et al. 2011) of breast cancer cells. Paradoxically, several studies have reported loss of YAP expression in human breast tumors as well as a correlation between improved patient survival and increased YAP mRNA levels, thereby implicating YAP as a breast cancer tumor suppressor (Matallanas et al. 2007; Yuan et al. 2008). Consistent with the latter view, it was shown that RNAi knockdown of YAP in breast cell lines suppressed anoikis, increased migration and invasiveness, and enhanced tumor growth in nude mice (Yuan et al. 2008). Reconciling these contrasting findings presents a challenge, especially given the heterogeneity of the genetic makeup of the tumor cells.

In this study, we used mouse genetics to interrogate Hippo signaling in the mammary gland in vivo. We show that the Hippo pathway is genetically dispensable in virgin mammary glands but specifically required during pregnancy, when mammary epithelia undergo rapid growth and terminal differentiation. In this context, hyperactivation of YAP fails to induce hyperplasia but results in a failure of mammary epithelial cells to undergo terminal differentiation. Interestingly, although inactivation of YAP causes no obvious defects in virgin mammary glands, it potently suppresses tumor growth and metastasis in a mouse model of oncogene-induced breast cancer. Our studies uncover a stage-specific requirement for Hippo signaling in mammary development and implicate YAP as a promising drug target for breast cancers.

\section{Results and Discussion}

Mammary epithelium undergoes several developmental phases, including ductal elongation in the virgin gland; proliferation, differentiation, and the formation of lobuloalveolar structures during pregnancy; and massive epithelial cell death in post-lactation-induced involution (Richert et al. 2000; Brisken and Rajaram 2006; Watson and Khaled 2008). We first examined the temporal and spatial patterns of YAP expression by immunohistochemistry. YAP protein was detected in cap cells and body cells in virgin terminal end buds (Supplemental Fig. S1A,B). In virgin mammary ducts, YAP protein was detected in

(C) 2014 Chen et al. This article is distributed exclusively by Cold Spring Harbor Laboratory Press for the first six months after the full-issue publication date (see http://genesdev.cshlp.org/site/misc/terms.xhtml). After six months, it is available under a Creative Commons License (Attribution-NonCommercial 3.0 Unported), as described at http:// creativecommons.org/licenses/by-nc/3.0/. 
luminal and myoepithelial cells (Supplemental Fig. S1C). The subcellular localization of YAP in the virgin glands differed in these cell types: Myoepithelial and cap cells showed prominent nuclear YAP accumulation, while the luminal and body cells displayed a more diffuse localization of YAP throughout the cytoplasm and nucleus (Supplemental Fig. S1A-C). In pregnant mammary glands, proliferating alveolar cells showed elevated YAP protein levels and prominent nuclear YAP (Supplemental Fig. S1D). During lactation, YAP staining in the alveoli was significantly decreased, with only a few scattered cells positive for YAP (Supplemental Fig. S1E). The involuted mammary glands showed YAP expression similar to that of virgin mammary glands, with prominent nuclear YAP staining detected in myoepithelial cells and much less detected in luminal cells (Supplemental Fig. S1F). The dynamic changes of YAP expression suggest that the Hippo signaling pathway may be required for mammary gland development in the pregnancylactation cycle.
To dissect the role of Hippo signaling in mammary development, we engineered mice with mammary-specific loss-of-function or gain-of-function of YAP by crossing floxed alleles of Yap or Sav1 (encoding a well-established tumor suppressor and negative regulator of the YAP oncoprotein) with the MMTV-Cre recombinase (Wagner et al. 1997). Successful deletion of Yap or Sav1 was confirmed, and the expression of the Yap paralog Taz was unchanged in the Yap-deficient mammary gland (Supplemental Fig. S2A-E). MMTV-Cre; Yap flox/flox and MMTV-Cre; Sav1flox/flox mice were born with the expected Mendelian ratio. Examination of Yap- or Sav1-deficient mammary glands from 6- and 8-wk-old virgin mice by whole mounts and paraffin sections revealed no defects in terminal end bud formation, ductal growth, or ductal branching (Fig. 1A-C; Supplemental Fig. S3). Similarly, transgenic overexpression of YAP did not perturb mammary gland morphology in virgin mice (see below). Thus, the Hippo pathway appears largely dispensable in the virgin mammary glands.
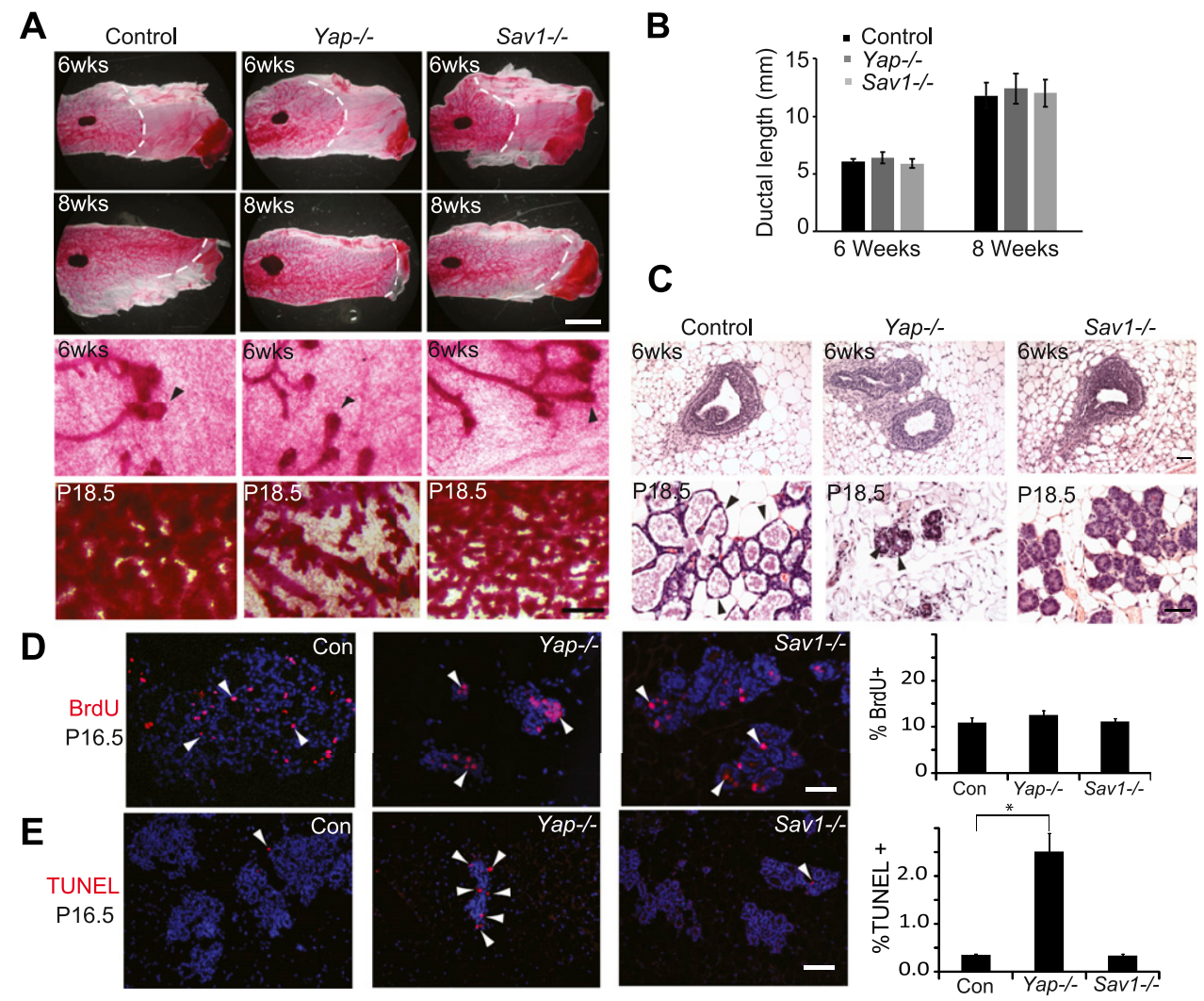

Figure 1. Impaired development in Yap-deficient and Sav1-deficient mammary glands. (A) Whole-mount staining of control (MMTV-Cre only), Yap-deficient, and Sav1-deficient mammary glands. (Top two rows) Low-magnification view of 6- and 8-wk virgin glands. Note the similar appearance and ductal invasion in each genotype. Bar, 5mm. (Bottom two rows) High-magnification view of 6-wk virgin and P18.5 glands. Note the normal appearance of terminal end buds of $\mathrm{Yap}^{-/-}$or Sav1 ${ }^{-/-}$virgin glands. Also note the greatly reduced lobuloalveolar structures of Yap-deficient glands and the tightly packed and undistended alveoli of Sav1-deficient glands in P18.5 glands. Bar, $500 \mu \mathrm{m}$. $(B)$ Quantification of ductal invasion in 6-and 8-wk virgin glands, measured as the distance between the distal end of the lymph node and the distal end of the longest duct. Data are mean $\pm \mathrm{SEM} ; n=3 . P>0.05, t$-test. $(C) \mathrm{H} \& \mathrm{E}$ staining of control (MMTV-Cre only), Yap-deficient, and Sav1-deficient mammary glands from 6-wk virgin and P18.5 glands. Note the normal appearance of terminal end buds in Yap ${ }^{-/-}$or Sav1 ${ }^{-1-}$ virgin glands. In P18.5 animals, fewer ducts and lobuloalveoli were present in Yap-deficient glands, and the remaining lobuloalveoli were significantly smaller and had fewer cells per alveolus compared with control. Sav1-deficient lobuloalveoli were undistended but contained a normal number of cells per alveolus. Also note the presence of lipid droplets (arrowheads) in control and Yap mutant glands but not in Sav1 mutant glands at P18.5. Bar , 50 $\mu \mathrm{m}$. (D) BrdU analysis of P16.5 glands. Arrowheads mark selected BrdU-positive cells. Note the similar number of BrdU-positive cells in each genotype. The graph shows mean \pm SEM, $n=5 . P>0.05, t$-test. Bar, $100 \mu \mathrm{m}$. (E) TUNEL analysis. Arrowheads mark examples of TUNEL-positive cells. Note the significant increase of TUNEL-positive cells in Yap-deficient, but not Sav1-deficient, mammary glands. The graph shows mean \pm SEM; $n=5$. $\left(^{\star}\right) P<0.05, t$-test. Bar, $100 \mu \mathrm{m}$. 
In contrast to its genetic dispensability in virgin glands, examination of Yap-deficient mammary glands at 16.5 and $18.5 \mathrm{~d}$ of pregnancy (P16.5 and P18.5, respectively) revealed significantly reduced alveolar structures (Fig. 1A,C; Supplemental Fig. S3). A similar hypoplasia was observed in organoid cultures of Yap-deficient mammary epithelia (Supplemental Fig. S4), supporting a cell-autonomous role for YAP in promoting mammary epithelial growth and/or survival during pregnancy. To understand the cellular mechanism underlying the Yap mutant phenotypes, we analyzed cell proliferation and apoptosis by BrdU and TUNEL staining. Compared with the control, Yapdeficient glands showed no alteration in cell proliferation (Fig. 1D) but a significant increase in apoptosis (Fig. 1E). Strikingly, despite hypoplasia, the Yap-deficient alveoli were positive for P-Stat5 and Npt2b, two markers that are normally expressed in terminally differentiated mammary alveolar cells (Fig. 2A,B; Liu et al. 1996; Long et al. 2003; Shillingford et al. 2003), suggesting that YAP is required for cell survival but not terminal differentiation of mammary alveoli. Consistent with this finding, we observed accumulation of cytoplasmic lipid droplets, a hallmark



Figure 2. Loss of Sav1 leads to defects in terminal differentiation of mammary glands. $(A-C)$ P18.5 mammary glands were analyzed for P-Stat5 (green) and smooth muscle actin (SMA, red) $(A), \mathrm{Npt2b}$ (green) and SMA (red) $(B)$, and H\&E staining $(C)$. Note the presence of P-Stat5 and Npt2b staining in $\mathrm{Yap}^{-1-}$ and Sav1 ${ }^{-1-} \mathrm{Yap}^{-1-}$ glands and its absence in Sav1 $1^{-1-}$ glands. Bars, $20 \mu \mathrm{m}$. (D) Control (MMTV-Cre only), Yap-deficient, and Sav1-deficient mammary glands at P13.5 were stained for AQP5 (red) plus DAPI (blue) or NKCC1 (green) plus SMA (red). Note the presence of AQP5 and NKCC1 signals (arrowheads) in virgin but not P13.5 glands in all genotypes. Bar, $20 \mu \mathrm{m}$. of terminal mammary differentiation, in Yap-deficient alveoli in vivo (Fig. 1C) or in cultured mammary organoids in vitro (Supplemental Fig. S4). Thus, while YAP is largely dispensable in the virgin mammary glands, it is required for the survival of mammary epithelial cells during pregnancy.

Next, we analyzed Sav1-deficient mammary glands at P16.5 and P18.5. Unlike normal alveoli in late pregnancy, which showed a distended morphology and abundant cytoplasmic lipid droplets, Sav1 mutant glands appeared less differentiated, as they contained tightly packed alveoli without lipid droplets (Figs. 1A,C, 2C; Supplemental Fig. S3). Consistent with the histological defects, the differentiation markers P-Stat 5 and Npt $2 b$ were completely absent in Sav1-deficient glands in late pregnancy (Fig. 2A,B). A similar defect in lipid droplets accumulation was observed in organoid cultures of Sav1-deficient mammary epithelial cells (Supplemental Fig. S4). Despite their differentiation defects, the Sav1-deficient glands exhibited alveolar cell numbers similar to those of control glands, which distinguished them from the alveolar hypoplasia observed in the Yap-deficient mammary glands (Figs. 1A,C, 2A-C). Indeed, Sav1 mutant glands showed normal levels of cell proliferation and apoptosis (Fig. 1D,E).

We noted that the Sav1-deficient alveoli showed normal expression of Keratin 8 and Keratin 14, markers for the luminal and myoepithelial layers, respectively, suggesting that these two cell types are specified correctly (Supplemental Fig. S2F; Guelstein et al. 1988; Wetzels et al. 1989). To further evaluate the differentiation defects, we examined two markers that are normally expressed in virgin mammary glands but are down-regulated at earlier stages of pregnancy. AQP5 (aquaporin5) is present on the apical membrane of ductal cells in virgin glands, and its expression is normally turned off at early pregnancy (Shillingford et al. 2003). $\mathrm{NKCC} 1$ is a $\mathrm{Na}-\mathrm{K}-\mathrm{Cl}$ cotransporter that is enriched on the basolateral membrane of ductal mammary epithelial cells in virgin glands, and its expression is normally turned off in developing alveoli and ductal structures at mid pregnancy (Shillingford et al. 2003). In Sav1-deficient mammary glands, AQP5 and NKCC1 were down-regulated by mid-pregnancy as in control glands (Fig. 2D). Thus, the differentiation defects of Sav1 mutant mammary glands are specific to late pregnancy but not early/mid-pregnancy.

The results presented so far suggest that Sav1 and Yap are dispensable in virgin mammary glands but are required specifically during pregnancy, with loss of Sav1 and Yap affecting mammary cell differentiation and survival, respectively. To better understand the relationship between Sav1 and Yap in mammary development, we generated Sav1; Yap double-mutant mammary glands. As in the respective single mutants, we found no abnormalities in the double-mutant virgin mammary glands. In late pregnancy, however, the Sav1; Yap double-mutant mammary glands showed phenotypes similar to those of 
Yap mutant glands: They both contained significantly reduced alveolar structures that were positive for the late differentiation markers P-Stat5 and Npt2b (Fig. 2A-C). Thus, loss of Yap could rescue the terminal differentiation defects of the Sav1 mutant, suggesting that YAP is a critical mediator of Sav1 in alveolar differentiation. Consistent with this view, Sav1 mutant mammary glands showed decreased phosphorylation at the Hippo-responsive $\mathrm{YAP}^{\mathrm{S} 112}$ phosphorylation site (Supplemental Fig. S2D).

Our findings support a model in which Hippo signaling, via inhibition of YAP, promotes terminal mammary differentiation during pregnancy. This model predicts that overexpression of YAP may result in differentiation defects similar to loss of Sav1. We tested this hypothesis through transgenic overexpression of human YAP under the control of a tetracycline response element (TRE) (Dong et al. 2007). Crossing the TRE-YAP strain with the mammaryspecific MMTV-rtTA driver (Gunther et al. 2002) allowed mammary-specific YAP overexpression in a doxycyclineregulated manner (Supplemental Fig. S5A). Interestingly, upon doxycycline feeding starting at birth, the MMTVrtTA; TRE-YAP mice showed patchy expression of the transgene, effectually creating mosaic mammary glands in which YAP-overexpressing cells could be compared sideby-side with wild-type neighbors without YAP overexpression (Supplemental Fig. S5B). We detected no difference between YAP-overexpressing and nonoverexpressing mammary ducts in virgin glands (Supplemental Fig. S5B). However, in late pregnancy, the alveoli with YAP overexpression, but not those without YAP overexpression, failed to undergo terminal differentiation: They showed an undistended morphology (Fig. 3B) and lacked differentiation markers such as P-Stat5, Npt2b, and $\beta$-casein (Fig. 3D-F). The similar differentiation defects exhibited by the YAP transgenic glands and the Sav1-deficient glands are consistent with the genetic epistasis analysis presented earlier, further implicating YAP as a critical mediator of Hippo signaling in alveolar differentiation.

The MMTV-rtTA; TRE-YAP mice also allowed us to examine the effect of direct YAP overexpression on cell proliferation. Analysis of YAP-overexpressing and nonoverexpressing alveoli revealed no significant difference (Supplemental Fig. S5C), suggesting that, as in Sav1deficient glands (Fig. 1D,E), YAP overexpression did not affect mammary cell proliferation. Consistent with these findings, by 18 mo of age, no tumors or hyperplasia were observed in Sav1-deficient or YAP transgenic mammary glands from both nulliparous and multiparous animals ( $n>10$ for each genotype/parity group). Thus, unlike many other tissues such as liver and intestine, activation of YAP alone is insufficient to drive hyperplasia in mammary epithelia.

Given the unexpected finding that YAP activation is insufficient to induce mammary hyperplasia, we investigated whether YAP is required in breast tumorigenesis using a well-characterized mouse model of oncogeneinduced breast cancer. Expression of the polyoma middle $\mathrm{T}$ antigen (PyMT) under the control of the MMTV promoter results in early onset mammary tumors and lung metastases in virgin mice (Guy et al. 1992). Interestingly, PyMT-induced mammary tumors showed increased expression of YAP (Fig. 4A) and TAZ (Supplemental Fig. S6A). To examine whether YAP contributes to PyMT-induced breast cancer, we inactivated YAP by introducing $M M T V$ Cre; Yap flox/flox into the PyMT background. PyMT virgins
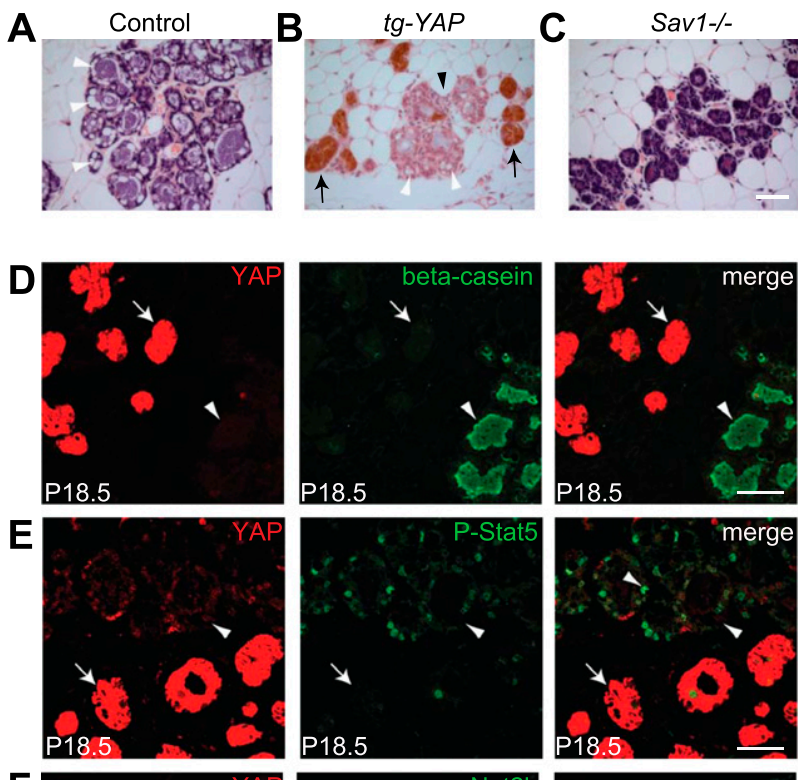

$\mathbf{F}$
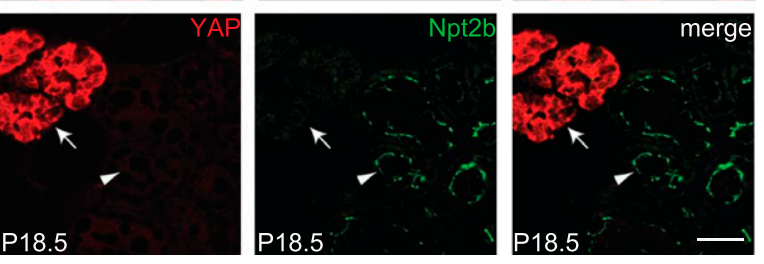

Figure 3. YAP overexpression causes terminal differentiation defects similar to loss of Sav1. (A) H\&E staining of a wild-type P18.5 mammary gland. Note the distended alveolar morphology and the cytoplasmic accumulation of lipid droplets (arrowheads). (B) A P18.5 MMTV-rtTA; TRE-YAP mammary gland stained for YAP (brown) and counterstained by H\&E. Note the mosaic pattern of YAP transgene induction, showing areas with (arrows) and without (arrowhead) YAP overexpression. Also note the nondistended morphology of YAP-overexpressing alveoli (arrow), while the neighboring nonoverexpressing alveoli (black arrowhead) showed distended morphology and cytoplasmic lipid droplets (white arrowheads). (C) H\&E staining of a P18.5 Sav1 mammary gland. Note the undistended morphology and the absence of cytoplasmic lipid droplets. Bar, $50 \mu \mathrm{m}$. $(D-F)$ P18.5 MMTV-rtTA; TRE-YAP transgenic mammary glands stained for YAP (red) and $\beta$-casein $(D), \mathrm{P}$-Stat5 $(E)$, or Npt2b $(F)$. Note the absence of these differentiation markers (green) in YAP-overexpressing alveoli (arrow) and the presence of these markers in nonoverexpressing alveoli (arrowhead). Bar, $20 \mu \mathrm{m}$.

developed palpable mammary tumors starting at $8 \mathrm{wk}$ of age, with $50 \%$ of virgins showing tumors by 12 wk and $100 \%$ of virgins showing tumors by $22 \mathrm{wk}$ of age $(n=33)$. In contrast, palpable tumors first appeared in the Yapdeficient PyMT virgins at 17 wk of age, with $50 \%$ and $100 \%$ tumor incidence occurring at 25 and 29 wk of age, respectively $(n=20)$ (Fig. 4B). Examination of 15 -wk-old Yap-deficient PyMT glands (before the detection of palpable tumors) revealed largely normal mammary epithelia containing a few focal hyperplasia, whereas age-matched PyMT glands showed widespread mammary hyperplasia and neoplasia (Fig. 4C). Consistent with suppression of tumor growth, phosphorylated histone $\mathrm{H} 3$ (PH3) and cleaved caspase-3 staining revealed decreased proliferation and increased apoptosis in the Yap-deficient PyMT glands compared with the PyMT glands (Fig. 4D). We also examined lung metastases in mice with end-stage tumors (at 22-29 wk of age). The incidence of lung metastases in the Yap-deficient PyMT 

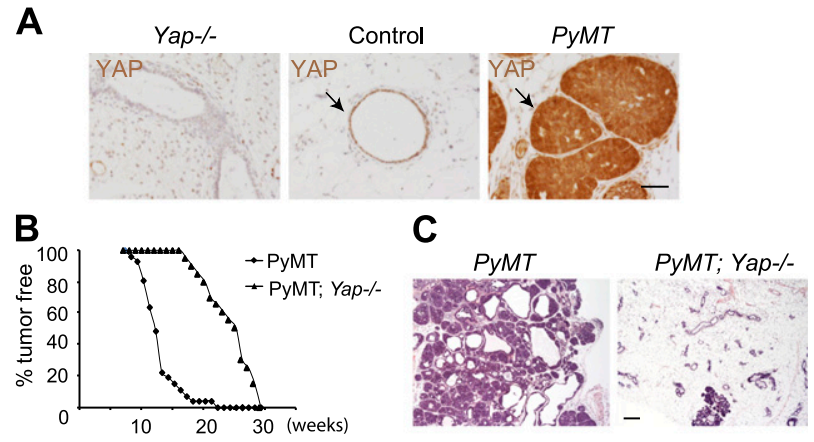

D
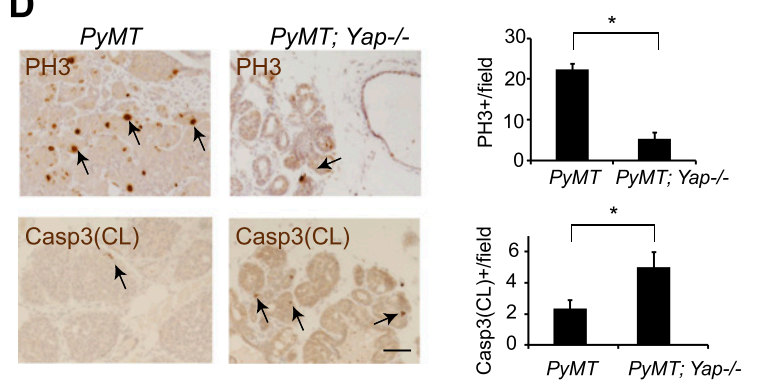

Figure 4. Loss of YAP suppresses PyMT-induced tumor growth. (A) Twelve-week-old Yap-deficient, control, and PyMT virgin glands were stained for YAP. Note the absence of YAP staining in the Yap-deficient mammary epithelia and elevated YAP expression in the PyMT mammary tumors (arrows). Bar, $50 \mu \mathrm{m}$. (B) Fraction of mammary pads free of palpable tumors in the PyMT (black diamond; $n=33$ ) and Yap-deficient PyMT (black triangle; $n=20$ ) virgin mice. (C) H\&E staining of 15-wk-old $P y M T$ and Yap-deficient $P y M T$ virgin glands. Note the significant suppression of mammary ductal hyperplasia in the Yap-deficient PyMT gland. Bar, $200 \mu \mathrm{m}$. (D) Analysis of cell proliferation and cell death in 15-wk-old PyMT and Yap-deficient PyMT virgin glands. Quantification is shown in the graphs. Note decreased cell proliferation and increased apoptosis in the Yapdeficient PyMT mice. $\left(^{\star}\right) P<0.05, t$-test. Bar, $50 \mu \mathrm{m}$.

mice (one out of 11) was significantly reduced compared with that in the PyMT mice (nine out of 17) (Supplemental Fig. S6B).

The genetic requirement for YAP in oncogene-induced breast cancer in mice prompted us to explore YAP inhibition as a means to suppress the growth of human breast cancer cells. For this purpose, we took advantage of verteporfin (VP), a recently reported YAP inhibitor that interferes with the physical association between YAP and its obligatory DNA-binding transcription factor partner, TEAD/TEF (Liu-Chittenden et al. 2012). A collection of eight commonly used human breast cancer lines with varying levels of YAP expression (Supplemental Fig. S7) were treated with VP at different concentrations (Fig. 5). Interestingly, cell lines with higher YAP expression levels were generally more sensitive to VP than cell lines with lower YAP expression levels. For example, T47D, which has the highest YAP expression levels, was fully inhibited by $1 \mu \mathrm{M}$ VP, whereas BT-483, which has the lowest YAP expression levels, was inhibited only by $10 \mu \mathrm{M}$ VP (Fig. 5). Thus, YAP inhibitors may be particularly effective against breast cancers driven by high levels of oncogenic YAP activity.

In summary, our studies provided unexpected insights into Hippo signaling in mammary development and tumorigenesis. First, in contrast to its potent oncogenic activity in cultured mammary epithelial cells, hyperac- tivation of YAP alone is insufficient to drive oncogenic growth in the normal mammary epithelia. Thus, it is likely that YAP-induced oncogenic growth requires cooperating genetic lesions. Consistent with this view, the YAP amplicon in mammary tumors was isolated from a genetic background bearing loss of Brca1 and p53 tumor suppressor genes (Overholtzer et al. 2006). Second, the Hippo pathway is dispensable in virgin glands but specifically required during pregnancy, when loss of YAP causes apoptosis of mammary epithelial cells, and hyperactivation of YAP results in a failure of terminal differentiation. This temporally restricted role of Hippo signaling is consistent with the dynamic changes of YAP expression during pregnancy, when mammary epithelial cells undergo tremendous expansion and differentiation. Of note, a recent study showed that YAP is dispensable for intestinal development but required in regenerating intestinal epithelia, which exhibit dynamic changes of YAP protein levels (Cai et al. 2010). Thus, Hippo signaling may be particularly important under conditions of rapid tissue growth and morphogenesis.

Our findings that loss of YAP results in apoptosis of mammary epithelial cells during pregnancy and that YAP is required for oncogene-induced breast cancers support an oncogenic rather than a tumor suppressor function of YAP in mammary tumorigenesis. The profound defect in alveolar differentiation upon Sav1 inactivation or YAP overexpression suggests that dysfunction of Hippo signaling may contribute to breast cancer genesis by blocking terminal differentiation, thereby providing a pool of immature cells that are not overproliferative per se but that are more susceptible to oncogenic transformation by cooperating genetic lesions. It is tempting to speculate that a YAP-induced differentiation block, as we uncovered in mammary development, may represent a more general mechanism by which the YAP oncoprotein promotes tumorigenesis in diverse mammalian tissue types. The dispensability of YAP in normal virgin glands, coupled with the genetic suppression of oncogene-induced breast
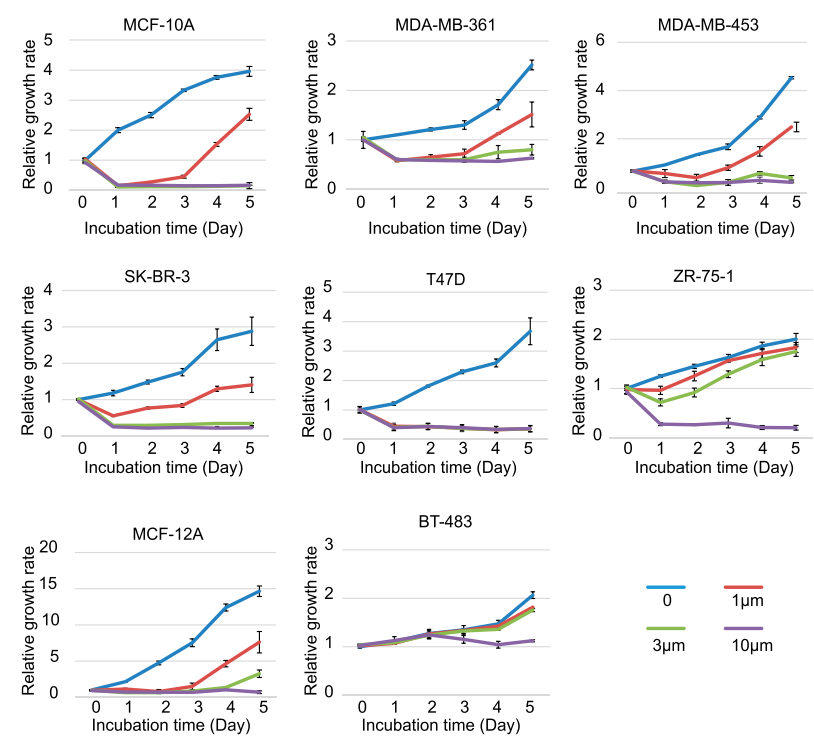

Figure 5. VP suppresses the growth of human breast cancer cell lines. Cells were seeded in 96-well plates with $0,1,3$, or $10 \mu \mathrm{M}$ VP. Cell number was measured at $0-5 \mathrm{~d}$. Note the differential sensitivity of the cell lines to VP. 
cancers by loss of YAP, suggests that YAP may be an ideal target for molecular targeted therapies against breast cancers.

\section{Materials and methods}

\section{YAP transgenic mice and Yap and Savl conditional knockout mice}

Pronuclear injection of linearized pTRE2-YAP transgene (Dong et al. 2007) was carried out by the Johns Hopkins Transgenic Core Facility. The pTRE2-YAP transgenic mice were identified by a PCR primer set (PS1, 5'-GTCCATGGTGATACAAGGGACATC-3'; PS2, 5'-GGCTGCCACCAA GCTAGATAAAG-3') that yielded a 700-base-pair product in positive lines and were maintained in a C57BL6 background. The MMTV-rtTA strain (FVB background) was kindly provided by Dr. Lewis C. Chodosh (Gunther et al. 2002). For YAP induction, transgenic and nontransgenic littermates (FVB/C57BL6 mixed background) were fed $2 \mathrm{mg} / \mathrm{mL}$ doxycycline (Sigma) in drinking water supplemented with $2.5 \%$ sucrose starting at birth.

Yaf ${ }^{f l o x}$ and Sav1 $1^{f l o x}$ mice have been published (Cai et al. 2010; Zhang et al. 2010). Mammary gland-specific knockout mice were generated by breeding Yap flox or Sav $1^{\text {flox }}$ mice with MMTV-Cre (line F) mice (Wagner et al. 1997) obtained from the National Cancer Institute Mouse Repository. Although this MMTV-Cre line was recently reported to show lactation defects resulting in the death of all pups before weaning (Robinson and Hennighausen 2011), in our colony, these mice (in a FVB/129/C57Bl6 mixed background) can rear $50 \%$ of pups to viable adults, and examination of mammary glands at $18.5 \mathrm{~d}$ of pregnancy reveals no histological difference between wild-type and MMTV-Cre mice. Therefore, we only examined mammary development at or earlier than P18.5, and mice bearing MMTVCre only were used as a control in all of our studies. For timing of the pregnant mice, noon of the day that the vaginal plug was observed was designated embryonic day 0.5 . All animal experiments were performed with protocols approved by the Johns Hopkins University Institutional Animal Care and Use Committee.

\section{Acknowledgments}

We thank J. Biber, L. Chodosh, M. Neville, and J. Turner for reagents; S. Htet, J. Cai, K. David, B. Huang, Y. Duan, F. Schozer, and C. Maltbie for technical assistance; and T. Lotan for advice on mammary histology. This work was supported in part by grants from the National Institutes of Health (R01 CA113795 to C.A.Z., and P50 CA088843 to A.J.E.), Flight Attendant Medical Research Institute (to C.A.Z.) and American Cancer Society (RSG12-141-01-CSM to A.J.E.). Q.C. is a recipient of Breast Cancer Research Post-doctoral Fellowship from the Department of Defense (BC093902). D.P. is an investigator of the Howard Hughes Medical Institute.

\section{References}

Brisken C, Rajaram RD. 2006. Alveolar and lactogenic differentiation. J Mammary Gland Biol Neoplasia 11: 239-248.

Cai J, Zhang N, Zheng Y, de Wilde RF, Maitra A, Pan D. 2010. The Hippo signaling pathway restricts the oncogenic potential of an intestinal regeneration program. Genes Dev 24: 2383-2388.

Chan SW, Lim CJ, Guo K, Ng CP, Lee I, Hunziker W, Zeng Q, Hong W. 2008. A role for TAZ in migration, invasion, and tumorigenesis of breast cancer cells. Cancer Res 68: 2592-2598.

Chen D, Sun Y, Wei Y, Zhang P, Rezaeian AH, Teruya-Feldstein J, Gupta S, Liang H, Lin HK, Hung MC, et al. 2012. LIFR is a breast cancer metastasis suppressor upstream of the Hippo-YAP pathway and a prognostic marker. Nat Med 18: 1511-1517.

Cordenonsi M, Zanconato F, Azzolin L, Forcato M, Rosato A, Frasson C, Inui $M$, Montagner $M$, Parenti AR, Poletti A, et al. 2011. The hippo transducer TAZ confers cancer stem cell-related traits on breast cancer cells. Cell 147: 759-772.

Dong J, Feldmann G, Huang J, Wu S, Zhang N, Comerford SA, Gayyed MF, Anders RA, Maitra A, Pan D. 2007. Elucidation of a universal sizecontrol mechanism in Drosophila and mammals. Cell 130: 1120-1133.

Guelstein VI, Tchypysheva TA, Ermilova VD, Litvinova LV, Troyanovsky SM, Bannikov GA. 1988. Monoclonal antibody mapping of keratins 8 and 17 and of vimentin in normal human mammary gland, benign tumors, dysplasias and breast cancer. Int J Cancer 42: 147-153.

Gunther EJ, Belka GK, Wertheim GB, Wang J, Hartman JL, Boxer RB, Chodosh LA. 2002. A novel doxycycline-inducible system for the transgenic analysis of mammary gland biology. FASEB J 16: 283-292.

Guy CT, Cardiff RD, Muller WJ. 1992. Induction of mammary tumors by expression of polyomavirus middle $\mathrm{T}$ oncogene: A transgenic mouse model for metastatic disease. Mol Cell Biol 12: 954-961.

Halder G, Johnson RL. 2011. Hippo signaling: Growth control and beyond. Development 138: 9-22.

Lamar JM, Stern P, Liu H, Schindler JW, Jiang ZG, Hynes RO. 2012. The Hippo pathway target, YAP, promotes metastasis through its TEADinteraction domain. Proc Natl Acad Sci 109: E2441-E2450.

Lei QY, Zhang H, Zhao B, Zha ZY, Bai F, Pei XH, Zhao S, Xiong Y, Guan KL. 2008. TAZ promotes cell proliferation and epithelial-mesenchymal transition and is inhibited by the hippo pathway. Mol Cell Biol 28: $2426-2436$.

Liu X, Robinson GW, Hennighausen L. 1996. Activation of Stat5a and Stat $5 \mathrm{~b}$ by tyrosine phosphorylation is tightly linked to mammary gland differentiation. Mol Endocrinol 10: 1496-1506.

Liu-Chittenden Y, Huang B, Shim JS, Chen Q, Lee SJ, Anders RA, Liu JO, Pan D. 2012. Genetic and pharmacological disruption of the TEADYAP complex suppresses the oncogenic activity of YAP. Genes Dev 26: 1300-1305.

Long W, Wagner KU, Lloyd KC, Binart N, Shillingford JM, Hennighausen L, Jones FE. 2003. Impaired differentiation and lactational failure of Erbb4-deficient mammary glands identify ERBB4 as an obligate mediator of STAT5. Development 130: 5257-5268.

Matallanas D, Romano D, Yee K, Meissl K, Kucerova L, Piazzolla D, Baccarini M, Vass JK, Kolch W, O'Neill E. 2007. RASSF1A elicits apoptosis through an MST2 pathway directing proapoptotic transcription by the $\mathrm{p} 73$ tumor suppressor protein. Mol Cell 27: 962-975.

Overholtzer M, Zhang J, Smolen GA, Muir B, Li W, Sgroi DC, Deng CX, Brugge JS, Haber DA. 2006. Transforming properties of YAP, a candidate oncogene on the chromosome 11q22 amplicon. Proc Natl Acad Sci 103: 12405-12410.

Pan D. 2010. The hippo signaling pathway in development and cancer. Dev Cell 19: 491-505.

Richert MM, Schwertfeger KL, Ryder JW, Anderson SM. 2000. An atlas of mouse mammary gland development. I Mammary Gland Biol Neoplasia 5: 227-241.

Robinson GW, Hennighausen L. 2011. MMTV-Cre transgenes can adversely affect lactation: Considerations for conditional gene deletion in mammary tissue. Anal Biochem 412: 92-95.

Shillingford JM, Miyoshi K, Robinson GW, Bierie B, Cao Y, Karin M, Hennighausen L. 2003. Proteotyping of mammary tissue from transgenic and gene knockout mice with immunohistochemical markers: A tool to define developmental lesions. I Histochem Cytochem 51: 555-565.

Wagner KU, Wall RJ, St-Onge L, Gruss P, Wynshaw-Boris A, Garrett L, Li M, Furth PA, Hennighausen L. 1997. Cre-mediated gene deletion in the mammary gland. Nucleic Acids Res 25: 4323-4330.

Watson CJ, Khaled WT. 2008. Mammary development in the embryo and adult: A journey of morphogenesis and commitment. Development 135: 995-1003.

Wetzels RH, Holland R, van Haelst UJ, Lane EB, Leigh IM, Ramaekers FC. 1989. Detection of basement membrane components and basal cell keratin 14 in noninvasive and invasive carcinomas of the breast. Am J Pathol 134: 571-579.

Yuan M, Tomlinson V, Lara R, Holliday D, Chelala C, Harada T, Gangeswaran R, Manson-Bishop C, Smith P, Danovi SA, et al. 2008. Yes-associated protein (YAP) functions as a tumor suppressor in breast. Cell Death Differ 15: 1752-1759.

Zhang N, Bai H, David KK, Dong J, Zheng Y, Cai J, Giovannini M, Liu P, Anders RA, Pan D. 2010. The Merlin/NF2 tumor suppressor functions through the yap oncoprotein to regulate tissue homeostasis in mammals. Dev Cell 19: 27-38.

Zhao B, Li L, Lei Q, Guan KL. 2010. The Hippo-YAP pathway in organ size control and tumorigenesis: An updated version. Genes Dev 24: $862-874$. 


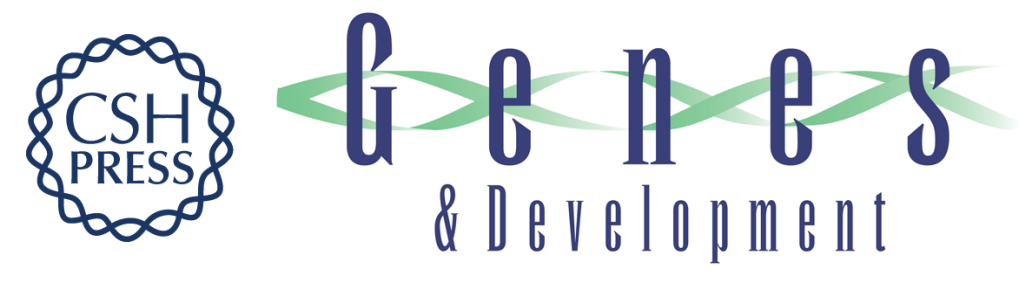

\section{A temporal requirement for Hippo signaling in mammary gland differentiation, growth, and tumorigenesis}

Qian Chen, Nailing Zhang, Ryan S. Gray, et al.

Genes Dev. 2014, 28:

Access the most recent version at doi:10.1101/gad.233676.113

\section{Supplemental http://genesdev.cshlp.org/content/suppl/2014/02/26/28.5.432.DC1 \\ Material}

References This article cites 27 articles, 11 of which can be accessed free at: http://genesdev.cshlp.org/content/28/5/432.full.html\#ref-list-1

Creative This article is distributed exclusively by Cold Spring Harbor Laboratory Press for the first Commons six months after the full-issue publication date (see

License http://genesdev.cshlp.org/site/misc/terms.xhtml). After six months, it is available under a Creative Commons License (Attribution-NonCommercial 3.0 Unported), as described at http://creativecommons.org/licenses/by-nc/3.0/.

Email Alerting Receive free email alerts when new articles cite this article - sign up in the box at the top Service right corner of the article or click here.

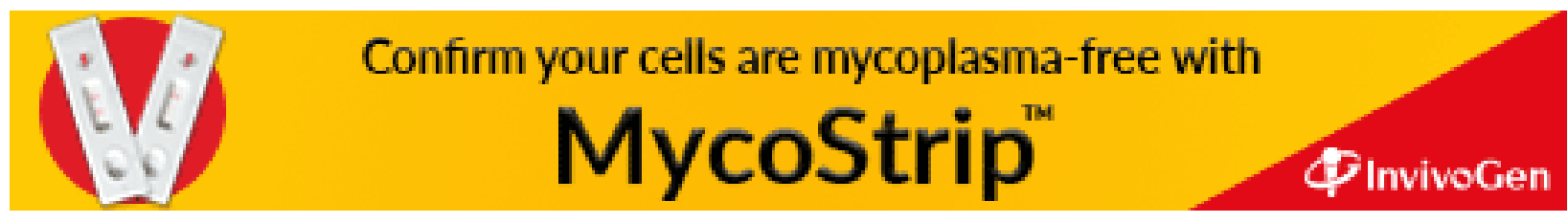

